\title{
El proyecto sobre el Área de Libre Comercio de Asia Pacífico en la competencia Sino-Estadounidense
}

The free trade area for the Asia Pacific project in the Sino-American competition

\author{
Juan José Ramírez Bonilla*
}

\section{Resumen}

En el artículo, es analizada la recuperación de la propuesta del APEC Business Advisory Council sobre la Free Trade Area for the Asia Pacific (Área de Libre Comercio de Asia Pacífico, ALCAP) por parte Xi Jinping, durante la reunión de APEC 2014; el tema es crucial para el futuro inmediato de la región del Pacífico, pues la iniciativa china va más lejos que APEC en el campo de los acuerdos vinculantes, al mismo tiempo que preserva APEC como un mecanismo para la contención de Estados Unidos. En ese sentido, el texto tiene en cuenta: a) La contradicción flagrante existente entre unas Metas de Bogor basadas en el "regionalismo abierto" y un acuerdo de comercio preferencial fundado en reglas que benefician a los signatarios, perjudicando a los no participantes. b) El contexto político actual del foro, determinado por la debilidad política de la administración Obama, resultante de las últimas elecciones legislativas, y por la situación crítica en que se encuentran las principales economías avanzadas. c) Los detalles de la impronta con que el gobierno chino ha marcado el proyecto del ALCAP.

Palabras clave:

- Orden económico internacional

- Negociaciones comerciales

- Integración económica

\section{Abstract}

In the article, it is analyzed the recovery of the proposed APEC Business Advisory Council on the Free Trade Area for the Asia Pacific (FTAAP) by Xi Jinping, during the meeting of APEC 2014; the issue is crucial for the immediate future of the Pacific region, as far as China's initiative goes beyond APEC in the field of binding agreements while preserving APEC as a mechanism for containment of the United States. In that sense, the text takes into account: a) The existing blatant contradiction between the Bogor Goals based on the "open regionalism" and the preferential trade agreement based on rules that benefit the signatories, hurting nonparticipants. $b$ ) The political context of the forum, determined by the political weakness of the Obama administration, resulting from the last legislative elections, and the critical situation in which are the major advanced economies. c) The details of the Chinese mark on the FTAAP project.

\section{Keywords:}

- International Economic Order

- Trade Negotiations

- Economic Integration

JEL: F02, F13, F15

\section{Introducción}

Durante la cumbre de APEC 2014, Xi Jinping ha llamado la atención de todo el mundo al anunciar que los "líderes económicos": "Decidimos comenzar y avanzar, de manera integral y sistémica, el proceso hacia la realización eventual del Área de Libre Comercio de Asia Pacífico (ALCAP) y respaldar la Hoja de ruta de Beijing para la contribución de APEC a la realización del ALCAP." Para

\footnotetext{
${ }^{1}$ APEC Secretariat, 2014 Leaders' Declaration. The 22nd APEC Ecomomic Leaders' Declaration-
} Beijing Agenda for an Integrated, Innovative and Interconnected Asia-Pacific, Beijing, APEC

\footnotetext{
* Centro de Estudios de Asia y África, El Colegio de México, A.C.jrami@colmex.mx
} 
Economía Informa núm. 389 noviembre - diciembre • 2014 " " " " " " " " " " -

comprender el alcance de la propuesta del Presidente de la República Popular China, debemos tener en cuenta: a) La tradición histórica de APEC en materia de liberalización comercial y financiera; a ese tema dedicamos la primera parte de nuestro texto, poniendo de realce la contradicción flagrante que existe entre unas Metas de Bogor basadas en el "regionalismo abierto" y un acuerdo de comercio preferencial fundado en reglas que benefician a los signatarios, perjudicando a los no participantes. b) El contexto político actual del foro, determinado por la debilidad política de la administración Obama, resultante de las últimas elecciones legislativas, y por la situación crítica en que se encuentran las principales economías avanzadas; dicho contexto será consecuentemente analizado en la segunda parte de nuestro trabajo. c) Los detalles de la impronta con que el gobierno chino ha marcado el proyecto del ALCAP y, en la tercera parte, destacamos los rasgos originales de la propuesta china, pues se orienta a ir más lejos que APEC en el campo de los acuerdos vinculantes, preservando APEC como un mecanismo para la contención de Estados Unidos.

\section{APEC y la liberalización comercial en una perspectiva histórica}

Han transcurrido 20 años desde que el Grupo de Personas Eminentes de APEC (GPE) propuso a los integrantes del foro liberar el comercio y las inversiones en Asia Pacífico. En 1994, el GEP planteaba una estrategia diametralmente opuesta a la pregonada por Xi Jinping, para lograr la liberalización comercial y financiera:

No proponemos la creación de un Área de Libre Comercio de Asia Pacífico. Nos pronunciamos por la creación de libre comercio en la región. Lo hacemos con el mayor énfasis posible sobre la [posibilidad] de alcanzar ese objetivo mediante la liberalización multilateral en el GATT. Más aun, nuestra propuesta para poner en práctica el concepto de APEC sobre el "regionalismo abierto" implica que la "región," en la cual el comercio y las inversiones libres resulten directamente de iniciativas de APEC, debe extenderse más allá de los límites geográficos de [los países] miembros de APEC... ${ }^{2}$

Secretariat, 11 de noviembre de 2014, http://www.apec.org/Meeting-Papers/Leaders-Declarations/2014/2014_aelm.aspx?p=1

${ }^{2}$ APEC Secretariat, Achieving the APEC Vision: Free and Open Trade in the Asia Pacific, Second Report of the Eminent Persons Group, Singapore, APEC Secretariat, 1994. 
La oposición enfática a un área de libre comercio de basaba en que cualquier acuerdo preferencial, por necesidad, discrimina todos los actores no participantes; así, para el GPE, el concepto del "regionalismo abierto" implicaba la supresión voluntaria y unilateral de todas las barreras arancelarias y no arancelarias, para permitir el libre flujo de bienes y de capitales, tanto entre los miembros del foro, como entre éstos y el resto del mundo. El GPE proponía adicionalmente que, antes de 1996, los participantes en APEC fijasen una fecha perentoria para poner en práctica su concepción del regionalismo abierto.

En 1994, en la segunda "reunión informal," en Bogor, Indonesia, los "líderes económicos de APEC" recogieron la propuesta del GPE y en su declaración estipularon:

...acordamos anunciar nuestro compromiso para alcanzar el objetivo de comercio e inversiones libres y abiertos en Asia-Pacifico, a más tardar en 2020. El ritmo para alcanzarlo tendrá en cuenta los niveles diferenciales de desarrollo entre las economías de APEC, con las economías industrializadas alcanzando el objetivo de comercio e inversiones libres y abiertos a más tardar en 2010 y, las economías en desarrollo, a más tardar en $2020 .^{3}$

A partir de entonces, las fechas perentorias para la supresión de las barreras al comercio y a las inversiones fueron conocidas como las Metas o la Agenda de Bogor. En 1994, los "líderes" también hicieron suyo el principio del "regionalismo abierto," propuesto por el GPE, cuando afirmaronn que "el resultado de la liberalización del comercio y las inversiones en Asia-Pacífico no será tan solo la reducción de barreras entre las economías de APEC, sino también entre las economías de APEC y las que no participan en el foro". ${ }^{4}$

En otros espacios, hemos tenido oportunidad de explicar cómo la liberalización del comercio y de las inversiones fue convertida en el fin último de APEC y cómo los mecanismos para lograr una liberalización progresiva del comercio y de las inversiones fueron suspendidos, debido a las dificultades derivadas de la crisis asiática de 1997, de la recesión estadounidense de 2001 y de la crisis financiera global de 2008-2009. ${ }^{5}$ En resumen, el agotamiento de los factores que hicieron posible la expansión económica global durante los

\footnotetext{
3 APEC Secretariat, 1994 Leaders' Declaration, Bogor Declaration-APEC Economic Leaders' Declaration of Common Resolve, Bogor, Indonesia, APEC Secretariat, 15 de noviembre de 1994, http://www.apec.org/Meeting-Papers/Leaders-Declarations/1994/1994_aelm.aspx 4 Idem.

5 Juan José Ramírez Bonilla, “APEC: pasado, presente... ¿y cuál futuro?,” en: Estudios de Asia y África. vol. 45. núm. 2. México D. F., El Colegio de México, 2010, pp. 423-449.
} 
Economía Informa núm. 389 noviembre - diciembre • 2014 " " " " " " " " " " -

años noventa del siglo pasado canceló las posibilidades de poner en práctica el "regionalismo abierto" pregonado por el GPE.

Por otra parte, el tránsito de la Comunidad Económica Europea a la Unión Europea (UE) sobre la base de un mercado y de una moneda comunes, así como la negociación y la puesta en práctica del Acuerdo de Libre Comercio de América del Norte (ALCAN), empujaron la evolución de la economía global en la senda opuesta a la aconsejada por los miembros del GPE: la negociación de acuerdos preferenciales. Las motivaciones en materia de política económica internacional cambiaron completamente de naturaleza: ante la discriminación resentida por los no participantes en esos proyectos de integración regional, los gobiernos excluidos buscaron negociar acuerdos preferenciales con la UE, con los miembros del alcan o entre ellos mismos. Así, la competencia se desplazó de los mercados a las mesas de negociaciones, para ver quién es capaz de establecer la red más amplia de acuerdos preferenciales. ${ }^{6}$

Esta nueva dinámica encaminó APEC en la senda opuesta a la indicada por el GPE: la creación no de una, sino de múltiples áreas de libre comercio; a pesar de que éstas siguen siendo mecanismos discriminatorios contra los no participantes, en APEC, los gobiernos pasan por alto que son obstáculos (stumbling blocks) para la consecución de comercio e inversiones libres y abiertos en la región, y los conciben acríticamente como medios (building blocks) para la liberalización comercial y financiera. En todo caso, la Organización Mundial de Comercio (OMC) registra 265 acuerdos comerciales regionales, los miembros de APEC participan en 124 (46.79\%) y 51 (19.24\%) son entre ellos mismos. Con agudeza, algunos autores han recurrido a la imagen del "tazón de espagueti," para describir el complejo entramado de las relaciones económicas reguladas por los acuerdos comerciales intergubernamentales. ${ }^{7}$

En este marco, Xi Jiping sorprende menos con la propuesta sobre la posible realización del Área de Libre Comercio de Asia Pacífico que con el olvido de la filosofía en materia de política económica subyacente en el concepto del "regionalismo abierto," propuesto por el GPE y adoptado por los "líderes" de APEC en sus primeras "reuniones informales". Una propuesta audaz hubiera sido que el presidente de la República Popular China anunciara una liberalización comercial y financiera unilateral, en beneficio de todas las economías

${ }^{6}$ Mireya Solís, "La economía política de Japón sobre acuerdos comerciales," en: Ramírez Bonilla, Juan José, Toledo Beltrán, Daniel y Uscanga, Prieto Carlos (coordinadores), Japón ante la nueva configuración de Asia del Pacífico. Proactividad y reactividad ante un orden internacional fluido, México, D. F., El Colegio de México, 2011.

${ }^{7}$ Robert Scoley, Preliminary Assessment of the Proposal for a Free Trade Area of the Asia-Pacific (FTAAP).An Issues Paper for the APEC Business Advisory Council (ABAC), s. 1., ABAC, 2004. 
del mundo; pero, como el horno no está para bollos, Xi Jiping ha debido plegarse al juego de la competencia político-diplomática prevaleciente en APEC, como veremos a continuación.

\section{El contexto político actual de la liberalización comercial y financiera}

A partir de 2001, el contexto político para la liberalización comercial y financiera promovida por APEC cambio de naturaleza; las causas de ese cambio fueron esencialmente dos: la primera fue la reorientación de la política exterior estadounidense hacia "la guerra contra el terrorismo internacional;" la segunda fue el surgimiento y la consolidación de la China como potencia regional.

En octubre de 2001, los "líderes" de APEC se reunieron en Shanghai, China, y su Declaración comenzaba señalando los dos grandes problemas del momento: las dificultades experimentadas en el plano de la economía global, debidas a la recesión que puso fin a la inusitada expansión de la economía estadounidense durante la década precedente, y los riesgos económicos derivados del "ataque terrorista sobre Estados Unidos".

Para responder a los "ataques terroristas sobre Estados Unidos," la primera administración George W. Bush optó por concentrar su política exterior en la "guerra contra el terrorismo internacional," obteniendo del Consejo de Seguridad y de la Asamblea General de las Naciones Unidas, de la Organización de Estados Americanos y de la Organización del Tratado del Atlántico Norte, la venia para encabezar dicha "guerra." Las presiones sobre los miembros de APEC para introducir el tema en la agenda del foro fueron evidentes; sin embargo, la vinculación artificial entre terrorismo e islam, establecida inicialmente por el gobierno estadounidense, fue entendida como una amenaza para la estabilidad interior de Brunei, China, Filipinas, Indonesia, Malasia, Singapur y Tailandia; debido a ello, los gobiernos asiáticos, encabezados por el anfitrión de APEC 2001, optaron por formular una declaración sobre el tema, indicando:

Los líderes se comprometen a prevenir y suprimir todas las formas de actos terroristas... en concordancia con la Carta de las Naciones Unidas y otras leyes internacionales, prometen poner en práctica las resoluciones 1368 y 1373 del Consejo de Seguridad fiel e inmediatamente, apoyan firmemente todos los esfuerzos para fortalecer el régimen internacional anti-terrorista,

\footnotetext{
8 APEC Secretariat, 2001 Leaders' Declaration, Shanghai Declaration-Meeting New Challenges in the New Century, Shanghai, China, APEC Secretariat, 21 de octubre de 2001, http://www.apec.org/ Meeting-Papers/Leaders-Declarations/2001/2001_aelm.aspx
} 
Economía Informa núm. 389 noviembre - diciembre • 2014 " " " " " " " " " " -

convocan para incrementar la cooperación tendiente a llevar ante la justicia a los perpetradores, y también invitan a firmar y ratificar todas las convenciones internacionales anti-terroristas... ${ }^{9}$

El mensaje era claro: evitar que el terrorismo fuese combatido mediante el terrorismo de Estado; para eso, la Declaración sobre el tema anteponía el derecho y las convenciones internacionales como el marco para prevenir y suprimir "todas las formas de actos terroristas." La administración Bush prosiguió con la "cruzada" antiterrorista, descuidando la relación con sus contrapartes de Asia del Pacífico.

La recesión estadounidense de 2001, permite comprender la actitud bivalente de las administraciones George W. Bush en materia de política económica internacional; por un lado, mantuvieron un discurso favorable al libre comercio; por otro, optaron por poner en práctica el proteccionismo relativo, subyacente en los mal denominados acuerdos de libre comercio, evitando los compromisos intergubernamentales con sus principales competidores económicos participantes en APEC. Como muestra el cuadro anexo, a las administraciones George W. Bush corresponde el mérito de haber firmado 10 de los 13 acuerdos comerciales notificados hasta ahora por el gobierno estadounidense a la OMC; teniendo en cuenta APEC, el gobierno estadounidense estableció cierto balance pues, de esos 10 acuerdos, cinco fueron establecidos con socios del foro y cinco con no participantes; en términos geográficos, privilegió la relación con América Latina (cuatro acuerdos comerciales), seguida por los vínculos con África del Norte y Medio Oriente (tres acuerdos), con Asia del Pacífico (dos acuerdos) y Oceanía (un acuerdo).

\footnotetext{
9 APEC Secretariat, Statement on Counter-Terrorism, Beijing, China, APEC Secretariat, 21 de octubre de 2001, http://apec.org/Meeting-Papers/Leaders-Declarations/2001/2001_aelm/statement_ on_counter-terrorism.aspx
} 


\section{Cuadro I}

Acuerdos comerciales entre Estados Unidos y socios "estratégicos"

\begin{tabular}{|c|c|c|c|c|}
\hline & & Firma & $\begin{array}{l}\text { Entrada } \\
\text { en vigor }\end{array}$ & $\begin{array}{l}\text { Tiempo entre } \\
\text { firma y entrada } \\
\text { en vigor }\end{array}$ \\
\hline Ronald Reagan & EEUU - Israel & $22-a b r-85$ & 19-ago-85 & 4 meses \\
\hline George Bush & ALCAN & 17-dic-92 & 01-ene-94 & 1 año 14 días \\
\hline \multirow[t]{5}{*}{ William Clinton } & EEUU - Jordania & $24-$ oct-00 & 17-dic-01 & 1 año 1 mes 23 días \\
\hline & EEUU - Singapur & 06-may-03 & 01-ene-04 & 6 meses 24 días \\
\hline & EEUU - Chile & 06-jun-03 & 01-ene-04 & 5 meses 24 días \\
\hline & EEUU - Australia & 18-may-04 & 01-ene-05 & 6 meses 13 días \\
\hline & EEUU - Marruecos & 15-jun-04 & 01-ene-06 & 1 año 6 meses 15 días \\
\hline \multirow{6}{*}{ George W. Bush } & EEUU - Bahréin & 15-sep-04 & 01-ago-06 & 1 año 11 meses 15 dias \\
\hline & EEUU - Omán & 19-ene-06 & 01-ene-09 & 2 años 11 meses 19 días \\
\hline & EEUU - Perú & $12-a b r-06$ & 01-feb-09 & 2 años 9 meses 12 días \\
\hline & EEUU - Colombia & 22-nov-06 & 15-may-12 & 5 años 6 meses 7 días \\
\hline & EEUU - Corea del Sur & 30-jun-07 & 15-mar-12 & 4 años 8 meses 15 días \\
\hline & EEUU - Panamá & 28-jun-07 & 31-oct-12 & 5 años 4 meses \\
\hline
\end{tabular}

El cuadro también permite ver los mecanismos propios del sistema político estadounidense: durante la primera administración George W. Bush el plazo transcurrido entre la firma y la entrada en vigor de un acuerdo, osciló entre seis meses, trece días y un año, once meses, quince días; para los acuerdos firmados durante la segunda administración, el plazo fue ampliado de dos años, nueves meses, doce días hasta cinco años, seis meses, siete días. Debido a esta peculiaridad, los acuerdos con Colombia, Corea del Sur y Panamá, aunque negociados por la administración George W. Bush, entraron en vigor durante la primera administración Barack H. Obama. Debemos pues tener en consideración que las diferencias en materia de política económica internacional entre las ramas ejecutiva y legislativa terminaron por entrabar los mecanismos regulatorios de la tímida apertura comercial hacia la región de Pacífico.

Esa timidez en materia de política económica generó un vacío en Asia del Pacífico, llenado rápidamente por el tándem Asociación de Naciones del Sureste de Asia (ANSEA)-China; en efecto, tan sólo dos semanas después de la 
Economía Informa núm. 389 noviembre - diciembre • 2014 " " " " " " " " " " -

reunión de APEC, en Shanghai, los jefes de Estado y del Gobierno de AnsEa y China se reunieron en Bandar Seri Begawan, Brunei, y anunciaron su voluntad conjunta de comprometerse en un Acuerdo marco sobre cooperación económica integral entre la ANSEA y la República Popular China y en el establecimiento de un Área de Libre Comercio ANSEA-China; ${ }^{10}$ la iniciativa sentó las bases de un orden institucional basado en las convenciones jurídicas de la ANSEA; el gobierno chino y posteriormente los gobiernos de Japón, Corea, Australia, Nueva Zelanda, India e, incluso, Brasil, han debido suscribir esas convenciones jurídicas para participar en ese orden institucional regional; el resultado ha sido el sistema radial de acuerdos comerciales, centrado en la ANSEA, con ramificaciones bilaterales que incluyen a cada uno de esos países (excepto Brasil).

En las postrimerías de la segunda administración George W. Bush, el gobierno estadounidense cobró consciencia del terreno político perdido y decidió, en enero de 2008, negociar con los gobiernos de Brunei, Chile, Nueva Zelanda y Singapur, su participación en el Trans-Pacific Strategic Economic Partnership (TPP), en operación desde el 28 de mayo de 2006. En noviembre de 2008, Australia, Vietnam y Perú decidieron participar en las negociaciones, sumándoseles Malasia, en octubre de 2010; en 2012, finalmente, Canadá, Japón y México completaron el grupo de negociadores del TPP-12. ${ }^{11}$

Las negociaciones del TPP-12 han sido secretas; pero, de acuerdo con la página electrónica de la Office of the US Trade Representative, veinte capítulos están bajo negociación: ${ }^{12}$ los sitios de internet con filtraciones sobre la agenda negociada se han multiplicado y dan cuenta de las dificultades, en múltiples campos, para establecer los consensos deseados. No es pues extraño que las negociaciones se hayan extendido más allá de noviembre de 2011, cuando la administración Obama detentó la presidencia pro tempore de APEC.

En todo caso, para los fines de nuestro texto, interesa destacar que las autoridades chinas han considerado la iniciativa estadounidense como una forma de aislar a China; por nuestra parte, consideramos que la amplia agenda del TPP también representa un complejo sistema técnico, cuya finalidad es proteger a

${ }_{10}$ ASEAN Secretariat, Framework Agreement on Comprehensive Economic Co-Operation between the Associations of Southeast Nations and the People's Republic of China, Phnom Penh, ASEAN Secretariat, Camboya, 4 de noviembre de 2002, http://www.asean.org/images/2013/economic/ afta/ACFTA/6-2002\%20-\%20Framework\%20Agreement $\% 20$ on $\% 20$ Comprehensive $\% 20$ Economic\%20Co-operation\%20between\%20ASEAN\%20and\%20China.pdf

11 Wikipedia, Trans-Pacific Partnership, http://en.wikipedia.org/wiki/Trans-Pacific_Partnership 12 Office of the United States Trade Representative, Outlines of the Trans-Pacific Partnership Agreement, Washington, D.C., Office of the United States Trade Representative, s.f. http://www.ustr. gov/about-us/press-office/fact-sheets/2011/november/outlines-trans-pacific-partnership-agreement. 
la economía estadounidense de la competencia asiática y, por extensión, a las economías participantes. Ante la iniciativa del TPP y como si fuera una partida de ajedrez, las autoridades chinas movieron sus piezas en el tablero regional y, el 20 de noviembre de 2012, teniendo como marco la Cumbre del Este de Asia (otro de los ámbitos políticos promovidos por la ANSEA), los diez gobiernos de la AnSEA, más los de Australia, China, Corea, India, Japón y Nueva Zelanda, decidieron poner en marcha las negociaciones del Regional Comprehensive Economic Partnership (RCEP).

El contraste entre las negociaciones del TPP y las del RCEP no puede ser mayor: mientras las primeras abarcan numerosos campos extra-comerciales y extra-financieros y se desarrollan sobre la base de los intereses económicos estadounidenses, las segundas tienen en cuenta "los diferentes niveles de desarrollo de los países participantes, el RCEP incluirá formas apropiadas de flexibilidad, incluyendo provisiones especiales y tratamiento diferencial, más flexibilidad adicional a los Estados miembro de ANSEA menos desarrollado, consistente con los ALC ANSEA +1 ". ${ }^{13}$ De hecho, la mención de los ALC ANSEA +1 hay que referirla de manera directa al ALC ANSEA +China, cuya experiencia sirve de base a la iniciativa del RCEP; en ese sentido, las negociaciones comerciales del ALC ANSEA+China fueron expeditas, pues fueron tomados en cuenta no sólo los niveles de desarrollo de los países negociadores, sino también los intereses de cada uno de ellos; así, cada uno pudo establecer una lista de productos sensibles y la desgravación para esos fue sometida a calendarios específicos.

Con la eventual culminación de las negociaciones del TPP y del RCEP, el tazón de espagueti mencionado en la primera parte de nuestro trabajo terminaría decantándose en estos dos mega-acuerdos comerciales, organizados, el primero, en torno a una potencia en declive y, el segundo, alrededor de una potencia en ascenso. Como sea, con la propuesta del RCEP, el gobierno chino, apoyado sobre la ANSEA, cuando menos logró equilibrar la relación de fuerzas en el escenario político de la región del Pacífico. La propuesta reciente sobre el Área de Libre Comercio de Asia del Pacífico (ALCAP), como veremos a continuación, vuelve a inclinar la balanza en favor de los asiáticos.

\footnotetext{
${ }^{13}$ asean Secretariat, Guiding Principles and Objectives for Negotiating the Regional Comprehensive Economic Partnership, s.1., ASEAN Secretariat, s. f., http://www.asean.org/images/2012/documents/Guiding\%20Principles $\% 20$ and $\% 20$ Objectives $\% 20$ for $\% 20$ Negotiating $\% 20$ the $\% 20$ Regional $\% 20$ Comprehensive \%20Economic\%20Partnership.pdf.
} 
Economía Informa núm. 389 noviembre - diciembre • 2014 " " " " " " " " " " -

\section{El ALCAP: un proyecto del ABAC, marcado con la impronta china}

Xi Jinping anunció la realización del AlCAP en el escenario más favorable: la cumbre de jefes de Estado y de Gobierno de APEC, realizada en la capital de China. Y, debemos añadir, el anunció también fue hecho en el momento más oportuno, en una coyuntura marcada por:

\section{La derrota electoral de medio término del Partido Demócrata}

- La pérdida de control sobre el Congreso estadounidense hace todavía más dificil que Barack H. Obama obtenga la autorización (fast track) para negociar el TPP.

- La autorización para negociar, si le fuera concedida, no significaría la entrada inmediata en vigor del TPP; como vimos, el lapso entre la firma de un acuerdo y su entrada en vigor se ha ampliado progresivamente, debido a las desavenencias entre el ejecutivo y el legislativo estadounidenses. Una vez firmado el TPP, el Congreso, dominado por los republicanos, podría exigir revisiones profundas del acuerdo negociado, como fue el caso del ALC Estados UnidosCorea.

- Los desacuerdos entre las partes negociadoras podrían profundizarse; pues, ante la posibilidad de alargar todavía más las negociaciones, la administración Obama podría otorgar mayores concesiones a sus contrapartes.

\section{La morosidad de las economías avanzadas}

- Japón, tan sólo cinco días después del anuncio del ALCAP, reconoció haber entrado en recesión, durante el tercer trimestre de $2014 .{ }^{14}$ La Abenomics ha sido reprobada por los hechos y tal parece que las dificultades de la economía nipona han orillado a la administración de Shinzo Abe a negarse a hacer concesiones sobre los sectores más sensibles, en las negociaciones del TPP. En términos políticos, Abe deberá enfrentar elecciones anticipadas y habrá que ver si las facciones políticas no le cobran la factura por haberse comprometido unilateralmente en las negociaciones del тPP.

- La Unión Europea, de acuerdo con las previsiones de Bruselas, a duras penas, registrará una tasa de crecimiento real del orden de 1.6\%, en 2014; en la zona euro, la situación será todavía más complicada, con una tasa de $1.2 \%{ }^{15}$ Por

\footnotetext{
${ }^{14}$ The New York Times, "Defying Expectations, Japan's Economy Falls into Recession," 16 de noviembre de 2014, http://www.nytimes.com/2014/11/17/business/international/defyingexpectations-japans-economy-shrinks-further.html

${ }^{15}$ lefigaro.fr, "Croissance: la charge de Washington qui redoute une "décennie perdue" en Europe,"13 de noviembre de 2014, http://www.lefigaro.fr/conjoncture/2014/11/13/20002-20141113ARTFIG00089-croissance-la-charge-de-washington-qui-redoute-une-decennie-perdue-en-europe. php.
} 
supuesto, habría que ver con detalle cuáles economías crecerán por encima o por debajo del promedio; pero, el futuro inmediato de la Unión no es muy halagüeño.

- $\quad$ Estados Unidos parece librar mejor los problemas; pero, durante 2014, la tendencia del crecimiento real es hacia la baja: $-2.1(1 \mathrm{er} \mathrm{T}), 4.6\left(2^{\circ} \mathrm{T}\right)$ y $3.5 \%$ $(3 \operatorname{erT}) \cdot{ }^{16}$

Ante un Obama políticamente disminuido y un mundo desarrollado en serios problemas, $\mathrm{Xi}$ Jinping no tuvo empacho en dirigirles sendos reproches velados en la Declaración 2014 de los "líderes de APEC." De manera inusual, Xi Jinping dejó de lado las fórmulas rituales sobre la adhesión a la OMC, para expresar "nuestra gran preocupación sobre el impasse [en que se encuentra] el Acuerdo para la Facilitación Comercial (AFC), se encuentra en un punto muerto y ha generado incertidumbre sobre otras decisiones [tomadas en] Bali. Estos acontecimientos han afectado la credibilidad de la función negociadora de la OMC". ${ }^{17}$ Por si eso no bastara y teniendo en mente las veleidades proteccionistas de los gobiernos de los países desarrollados, también refirmó "nuestro compromiso contra todas las formas de proteccionismo. Extendemos nuestro compromiso de no-acción [proteccionista] hasta finales de 2018 y reafirmamos nuestro compromiso para dar marcha atrás con cualquier medida proteccionista y distorsionante del comercio." ${ }^{18}$

La peculiaridades del proyecto ALCAP

Las condiciones fueron propicias para anunciar la puesta en marcha del proyecto del Área de Libre Comercio de Asia del Pacífico (ALCAP). Los comunicadores, en más de una ocasión, perdieron la perspectiva histórica, para atribuir a Beijing la pertenencia de la iniciativa; así, un presitigiado diario español anunciaba: "Pekín recibe el respaldo de la APEC para su zona de libre comercio."19 A todas luces, el artículo posesivo "su” es abusivo; pues el proyecto del ALCAP tiene su origen la iniciativa del APEC Business Advisory

\footnotetext{
${ }^{16}$ Bureau of Economic Analysis, Gross Domestic Product:Third Quarter 2014 (Advace Estimate), Washington, D.C., US Department of Commerce, 30 de octubre de 2014, http://www.bea.gov/newsreleases/national/gdp/2014/pdf/gdp3q14_adv.pdf.

${ }^{17}$ APEC Scretariat, 2014 Leaders' Declaration.

${ }^{18}$ Idem.

${ }^{19}$ LiyVidal, Macarenam “Pekín recibe el respaldo de la APEC para su zona de libre comercio," Madrid, El País, 11 de noviembre de 2014, http://internacional.elpais.com/internacional/2014/11/11/actualidad/1415724444_029371.html.
} 
Economía Informa núm. 389 noviembre - diciembre • 2014 " " " " " " " " " " -

Council (ABAC), en 2004, cuando las iniciativas para lograr una liberalización progresiva del comercio e inversiones fueron dejadas de lado, a causa de las dificultades económicas y políticas de los miembros de APEC. Más tarde, durante 2004 y 2005, ABAC y un grupo de trabajo del Pacific Economic Cooperation Council (PECC) prepararon un estudio de factibilidad sobre el ALCAP y fue sometido por los representates de ABAC a los "líderes de APEC," sin que haya tenido consecuencia alguna.

Ante la aproximación de la primera fecha perentoria de la Agenda de Bogor, en 2006, los "líderes" decidieron ocuparse del proyecto del ALCAP, comisionando a sus ministros para estudiar las modalidades y los caminos para lograr la integración regional en el marco de APEC. En 2010, en la reunión de Yokohama, Japón, los "líderes" formularon una declaración titulada Rutas hacia el ALCAP. El tema había sido incorporado de manera ritual en las declaraciones anuales de los "líderes," sin más.

Ahora, si bien es cierto que el proyecto del ALcAP no es chino, la modalidad propuesta para ponerlo en práctica lleva el sello Made in China; en efecto, la declaración de Xi Jingping envía de manera directa a la Hoja de ruta de Beijing para la contribución de APEC a la realización del ALCAP y, en ésta, podemos apreciar la originalidad de la estrategia china.

En nuestra opinión, el aspecto más importante es que el ALCAP no sería realizado como un proyecto dentro de APEC, sino en paralelo al foro. ${ }^{20}$ Las razones de peso son, cuando menos, tres: primero, el incumplimiento de la liberalización comercial y financiera por parte de los gobiernos de los países desarrollados, en 2010, minó la credibilidad de APEC; segundo, la competencia de los proyectos sobre el TPP y el RCEP, en el seno de APEC, generó percepciones negativas sobre la efectividad del foro como mecanismo de cooperación; tercero, cualquier tentativa de acuerdo comercial preferencial (incluidos el TPP y el RCEP) dentro de la estructura del foro va contra el espíritu original del mismo, basado en el consenso y las decisiones no vinculantes. La decisión de negociar el ALCAP en paralelo a APEC tiene como finalidad preservar el foro como una instancia de diálogo y de consulta, donde las potencias en competencia política pueden dirimir algunas de sus diferencias, buscando la mediación de los otros miembros del foro.

\footnotetext{
20 "The FTAAP will be realized outside of APEC, parallel with the APEC process. APEC should maintain its non-binding, voluntary cooperation principles in its contributions to the realization of the FTAAP," en APEC Secretariat, Annex A - The Beijing Roadmap for APEC's Contribution to the Realization of the FTAAP, Beijing, APEC Secretariat, 11 de noviembre de 2014. http://www.apec.org/ Meeting-Papers/Leaders-Declarations/2014/2014_aelm/2014_aelm_annexa.aspx?p=1.
} 
El segundo rasgo crucial de la propuesta es la diálectica establecida entre la nueva meta de Bogor (liberalización unilateral, comercial y financiera, en todas las economías de APEC, en 2020) y el ALCAP. Como señalamos, las metas de Bogor descansan sobre el concepto del "regionalismo abierto;" en consecuencia, las autoridades chinas se pronuncian por mantenerlas como un compromiso moral de los miembros de APEC; en contraste, el ALCAP siendo por necesidad un acuerdo vinculatorio y discriminatorio de los no signatarios, en opinión de los autores de la Hoja de ruta, ALCAP no puede substituir el objetivo de la Agenda de Bogor. Ahora bien, mientras el ALCAP tiene visos de ser puesto en práctica, habremos de esperar el 2020 para ver si, entonces sí, los miembros de APEC ponen en práctica el "regionalismo abierto."

Un tercer rasgo de la estrategia china es la idea de hacer converger sobre el ALCAP todas las iniciativas regionales. De nueva cuenta, sale a relucir la inefectividad del tazón de espagueti y la necesidad de simplificar, en la escala regional, el régimen de comercio administrado bajo un sólo mega-acuerdo. Suponemos que, en ese acuerdo, tendrán cabida todos los países de la ANSEA, aún cuando no todos sean miembros de APEC; si es así, entonces también cabe la posibilidad de que la Alianza del Pacífico sea englobada en el ALCAP.

Finalmente, es necesario volver sobre la función de APEC, se trata de una función renovada para "asistir a las economías de APEC interesadas, en participar en los procesos regionales y en prepararse para la realización del ALCAP;" en ese sentido, APEC "debe seguir proveyendo actividades efectivas en materia de cooperación económica y técnica que ayuden a las economías en desarrollo." En resumen, la propuesta china tiene una proyección hacia toda la región del Pacífico y uno no puede dejar de pensar en que se trata de la respuesta a las propuestas del TPP y del TTIP, encabezados por Estados Unidos, con el fin de excluir a China de esas iniciativas.

En efecto, con esos dos proyectos, la administración Obama ha dejado atrás la política de "contención de China" (es decir, la política basada en la inclusión de China en las instituciones multilaterales, regionales y bilaterales), para entablar una competencia por la supremacía político-diplomática en la región del Pacífico. La propuesta de Xi Jinping sobre la ALCAP es, a todas luces bivalente, pues, por un lado, otorga ventajas al gobierno chino en el marco en esa competencia político-diplomático y, por el otro, preserva APEC como un mecanismo para contener a un gobierno estadounidense deseoso de desentenderse de la política de "contención."

En el futuro inmediato, habremos de ver si la flexibilidad en materia de negociaciones y una agenda limitada a los temas clásicos del comercio y de 
Economía Informa núm. 389 noviembre - diciembre • 2014 " " " " " " " " " " -

las inversiones son suficientes para que el gobierno chino establezca un contrapeso a la política de redespliegue en Asia del Pacífico, practicada, no sin dificultades, por la administración Obama.

\section{Conclusiones}

La partida política entre los gobiernos chino y estadounidense se ha desarrollado hasta ahora en seis movimientos:

- Primero, el gobierno estadounidense dejó el campo libre en Asia del Pacífico, al privilegiar la "guerra contra el terrorismo internacional," como eje de su politica exterior.

- Segundo, el tándem ANSEA-China llenaron el vacío político y establecieron las bases de un orden institucional basado en una compleja red de acuerdos gubernamentales de cooperación, entre los cuales destacan los acuerdos comerciales bilaterales de la ANSEA con Australia, China, Corea, India, Japón y Nueva Zelanda.

- Tercero, para recuperar el terreno perdido en la región y aislar económicamente a China, el gobierno estadounidense ha intentado recuperar el proyecto del TPP y, ahora, 12 gobiernos miembros de APEC participan en unas negociaciones secretas, complicadas y, por ende, largas y desgastantes.

- Cuarto, para equilibrar la relación política de fuerzas en la región del Pacífico, el gobierno chino decidió responder a la iniciativa del TPP con el RCEP.

- Quinto, para completar el aislamiento en el campo de la diplomacia económica, el gobierno estadounidense optó por empujar a la Unión Europea a negociar el TTIP.

- Sexto, para lograr un nuevo re-equilibrio político, el gobierno chino ha lanzado ahora la negociación del ALCAP, poniendo en jaque a un gobierno estadounidense en dificultades, tanto domésticas como internacionales.

Todo indica que la administración Obama carece de tiempo para realizar un nuevo movimiento y tratar de neutralizar el ascendiente político de China en APEC, en particular, y en la región del Pacífico, en general. Pero, si pudiera hacerlo, a las autoridades chinas todavía les quedaría el recurso de solicitar su inclusión en el TPP, para ganar la partida.

Por lo pronto, con el anuncio de un acuerdo comercial con el gobierno coreano, las autoridades chinas han puesto en un serio predicamento a las japonesas; éstas, presionadas por los organizaciones de las grandes empresas trasnacionales, más pronto que tarde, habrán de ejercer presiones para parti- 
cipar en la nueva iniciativa de Xi Jinping. Acrecentando el momento político favorable a un gobierno chino, deseoso de invertir la relación de fuerzas mediante un sistema regional que permita "contener" a una potencia en declive y limitar los efectos perniciosos su pérdida de influencia política.

La región del Pacífico se encuentra -en consecuencia-, en un momento crucial y todos los países de la cuenca serán afectados de una u otra manera; hemos mencionado que, al considerar el proyecto del ALCAP en el márgen de APEC, los chinos apuntan hacia un megaproyecto que incluya todas las iniciativas en curso en la región. Por supuesto, los primeros destinatarios del mensaje son los miembros de la ANSEA que no participan en APEC y que podrían sumarse al Alcap. De este lado del océano, los socios de México en la Alianza del Pacífico también habrán de ser receptivos al mensaje y obligarán a los cuatro integrantes de la Alianza a presentar una posición común, so pena de que la onda de choque del ALCAP termine resquebrajando la unidad y el entusiasmo inicial de la Alianza.

\section{Bibliografía}

APEC Secretariat, 1994 Leaders' Declaration, Bogor Declaration-APEC Economic Leaders' Declaration of Common Resolve, Bogor, Indonesia, APEC Secretariat, 15 de noviembre de 1994, http://www.apec.org/Meeting-Papers/Leaders-Declarations/1994/1994_aelm.aspx

, 2001 Leaders' Declaration, Shanghai Declaration - Meeting New Challenges in the New Century, Shanghai, China, APEC Secretariat, 21 de octubre de 2001, http://www.apec.org/Meeting-Papers/Leaders-Declarations/2001/2001_ aelm.aspx

, Statement on Counter-Terrorism, Beijing, China, APEC Secretariat, 21 de octubre de 2001, http://apec.org/Meeting-Papers/Leaders-Declarations/2001/2001_ aelm/statement_on_counter-terrorism.aspx

, Achieving the Apec Vision: Free and Open Trade in the Asia Pacific, Second Report of the Eminent Persons Group, Singapore, APEC Secretariat, 1994 , 2014 Leaders' Declaration. The 22nd APEC Ecomomic Leaders' Declaration - Beijing Agenda for an Integrated, Innovative and Interconnected Asia-Pacific, Beijing, APEC Secretariat, 11 de noviembre de 2014, http://www.apec.org/ Meeting-Papers/Leaders-Declarations/2014/2014_aelm.aspx?p=1

, Annex A - The Beijing Roadmap for APEC's Contribution to the Realization of the fTAAP, Beijing, APEC Secretariat, 11 de noviembre de 2014, http://www. 


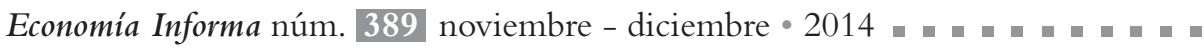

apec.org/Meeting-Papers/Leaders-Declarations/2014/2014_aelm/2014_aelm_ annexa.aspx?p=1

asean Secretariat, Guiding Principles and Objectives for Negotiating the Regional Comprehensive Economic Partnership, s.1., ASEAN Secretariat, s.f. http://www. asean.org/images/2012/documents/Guiding\%20Principles $\% 20$ and $\% 20$ Objectives $\% 20$ for $\% 20$ Negotiating $\% 20$ the $\% 20$ Regional $\% 20$ Comprehensive $\% 20$ Economic\%20Partnership.pdf

, Framework Agreement on Comprehensive Economic Co-Operation between the Associations of Southeast Nations and the People's Republic of China, Phnom Penh, Camboya, ASEAn Secretariat, 4 de noviembre de 2002, http://www.asean. org/images/2013/economic/afta/ACFTA/6-2002\%20-\%20Framework\%20 Agreement $\% 20$ on\%20Comprehensive $\% 20$ Economic\%20Co-operation $\% 20 \mathrm{be}-$ tween\%20ASEAN\%20and\%20China.pdf

Bureau of Economic Analysis, Gross Domestic Product: Third Quarter 2014 (Advace Estimate), Washington, D.C., US Department of Commerce, 30 de octubre de 2014, http://www.bea.gov/newsreleases/national/gdp/2014/pdf/gdp3q14_ adv.pdf.

lefigaro.fr, "Croissance: la charge de Washington qui redoute une "décennie perdue" en Europe," 13 de noviembre de 2014, http://www.lefigaro.fr/conjoncture/2014/11/13/20002-20141113ARTFIG00089-croissance-la-charge-de-washington-qui-redoute-une-decennie-perdue-en-europe.php.

Office of the United States Trade Representative, Outlines of the Trans-Pacific Partnership Agreement, Washington, D. C, Office of the US Trade Representative, s.f. http://www.ustr.gov/about-us/press-office/fact-sheets/2011/november/outlines-trans-pacific-partnership-agreement.

Ramírez Bonilla, Juan José: “Japón, Estados Unidos y la integración en la región del Pacífico: un paso adelante y otro atrás," en Cornejo, Romer (compilador), Asia Pacífico, 1994, México, D. F., El Colegio de México, 1994. , “APEC: pasado, presente... ¿y cuál futuro?,” en: Estudios de Asia y África. Vol. 45. no 2. México D. F. El Colegio de México, 2010. pp. 423-449.

Scoley, Robert: Preliminary Assessment of the Proposal for a Free Trade Area of the Asia-Pacific (ftAap). An Issues Paper for the APEC Business Advisory Council (ABAC), s.l., ABAC, 2004, http://www.pecc.org/resources/trade-and-investment-1/2027-preliminary-assessment-of-the-proposal-for-a-free-trade-areaof-the-asia-pacific.

Solís, Mireya: "La economía política de Japón sobre acuerdos comerciales," en: Ramírez Bonilla, Juan José, Toledo Beltrán, Daniel y Uscanga Prieto, Carlos 
(coordinadores), Japón ante la nueva configuración de Asia del Pacífico. Proactividad y reactividad ante un orden internacional fluido, México, D. F., El Colegio de México, 2011.

The New York Times, "Defying Expectations, Japan's Economy Falls into Recession," New York,16 de noviembre de 2014, http://www.nytimes.com/2014/11/17/ business/international/defying-expectations-japans-economy-shrinks-further. html.

Vidal Liy, Macarena: "Pekín recibe el respaldo de la APEC para su zona de libre comercio," Madrid, El País, 11 de noviembre de 2014, http://internacional.elpais. com/internacional/2014/11/11/actualidad/1415724444_029371.html.

Wikipedia, Trans-Pacific Partnership, http://en.wikipedia.org/wiki/Trans-Pacific_Partnership. 